\title{
Real-time statistical modelling of data generated from self-sensing bridges
}

1 F. Din-Houn Lau MSci (Hon), PhD Lecturer, Department of Mathematics, Imperial College London, London, UK; Group Leader, The Lloyd's Register Foundation Programme on Data-centric Engineering, The Alan Turing Institute, London, UK (corresponding author: dhl@imperial.ac.uk) (Orcid:0000-0003-1065-828X)

2 Liam J. Butler BASc, PhD, PEng Research Associate, The Lloyd's Register Foundation Programme on Data-centric Engineering; Group Leader, The Alan Turing Institute, London, UK; Cambridge Centre for Smart Infrastructure and Construction, Department of Engineering, University of Cambridge, Cambridge, UK
3 Niall M. Adams BSc (Hon), PhD, CStat Professor, Department of Mathematics, Imperial College London, London, UK; Data Science Institute, Imperial College London, London, UK

4 Mohammed Z. E. B. Elshafie BSc (Hon), MPhil (Cantab), PhD (Cantab)

Senior Lecturer, Cambridge Centre for Smart Infrastructure and Construction, Department of Engineering, University of Cambridge, Cambridge, UK; Visiting Professor, Department of Civil and Architectural Engineering, Qatar University, Doha, Qatar

5 Mark A. Girolami BSc (Hon), PhD, FRSE

Chair in Statistics, Department of Mathematics, Imperial College London, London, UK; The Lloyd's Register Foundation Programme on Data-centric Engineering, The Alan Turing Institute, London, UK
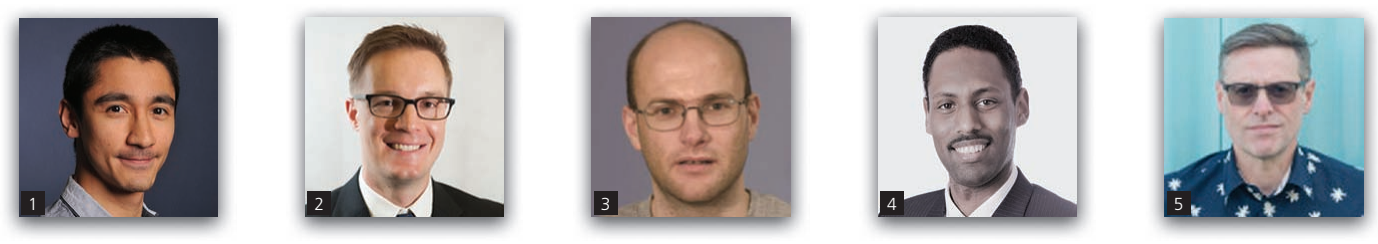

Instrumentation of infrastructure is changing the way engineers design, construct, monitor and maintain structures such as roads, bridges and underground structures. Data gathered from these instruments have changed the handson assessment of infrastructure behaviour to include data processing and statistical analysis procedures. Engineers wish to understand the behaviour of the infrastructure and detect changes - for example, degradation - but are now using high-frequency data acquired from a sensor network. Presented in this paper is a case study that models and analyses in real time the dynamic strain data gathered from a railway bridge which has been instrumented with fibre-optic sensor networks. The high frequency of the data combined with the large number of sensors requires methods that efficiently analyse the data. First, automated methods are developed to extract train passage events from the background signal and underlying trends due to environmental effects. Second, a streaming statistical model which can be updated efficiently is introduced that predicts strain measurements forward in time. This tool is enhanced to provide anomaly detection capabilities in individual sensors and the entire sensor network. These methods allow for the practical processing and analysis of large data sets. The implementation of these contributions will be essential for demonstrating the value of self-sensing structures.

\section{Notation}

$C_{b}$

$k$

$l$

$M_{s}$

$m_{t} \in \mathbb{R}$

$N\left(\mu, \sigma^{2}\right)$

$n_{t}$

$p_{s}$

$R^{2}$

S

$S$

$T$ scaled and centred observation for strain records moving window half-width

batch window half-width

statistical model for sensor $s$

(weighted) sum of $x_{t}$ values at time instance $t$

normal distribution with mean $\mu \in \mathbb{R}$ and variance

$\sigma^{2}>0$

(weighted) number of $x_{t}$ values observed at time instance $t$

$p$-value from one-step-ahead prediction of $Y_{t}^{(s)}$

coefficient of determination

total number of sensors

sensor

final time index $t \quad$ time index

$U_{s} \quad$ train passage event times of sensor $s$

$v \quad$ number of datapoints in batches

$w \quad$ number of datapoints used in a sliding window

$X^{2} \quad$ Fisher's $X^{2}$ statistic

$x_{t} \in \mathbb{R} \quad$ generic values at time instance $t$

$\bar{x}_{t} \quad$ (weighted) average of $x_{1}, \ldots, x_{t}$

$Y_{t}^{(s)} \quad$ random variable of the strain record for sensor $s$ at time $t$

$\widehat{Y}_{t}^{(s)} \quad$ one-step-ahead prediction for sensor s at time $t-1$

$Z_{b} \quad$ moving average of strain records

$\alpha \quad p$-value threshold, below which an anomaly is signalled

$\beta_{j} \quad$ unknown linear parameters in model $M_{\mathrm{s}}$ for $j=0,1$, 
$X^{2}$ threshold, above which an anomaly is signalled forgetting factor

wavelength at time instance $t: \mathrm{nm}$

photoelastic coefficient

$j$ th standard deviation for centred observations

\section{Introduction}

The potential of smart infrastructure to make more efficient use of existing and new assets has been estimated to be worth between $£ 2$ and $£ 4 \cdot 8$ trillion globally (Bowers et al., 2016). At the centre of this shift towards making assets smarter is the advance and maturity of sensor development and deployment. However, the introduction of vast sensor networks within infrastructure has already begun to inundate owners, engineers and maintainers with large volumes and varied quality, velocity and variety of data. From a civil engineering perspective, instrumentation of structures such as bridges has the potential to transform the design, construction, assessment and maintenance life cycle phases. One of the main challenges lies in the development of innovative methods for managing, processing, analysing and interpreting the data obtained from smart infrastructure assets. A collaboration between engineers at the Centre for Smart Infrastructure and Construction (CSIC) at the University of Cambridge and data scientists at the Lloyd's Register Foundation-funded Programme on Data-centric Engineering (DCE) at the Alan Turing Institute is focused on addressing this challenge. DCE is a synthesis of approaches to studying physical engineering assets which leverages physicsbased models which are updated based on measured data from the actual physical asset in operation and statistical (data-driven) models. This approach combines physical prior knowledge with empirical data, providing for the physical asset a 'digital twin' (Lau et al., 2018). The current study focuses on development of the statistical models. Statistical techniques offer a means of monitoring structural health which does not require knowledge of the structure's behaviour. Instead, such models can be used to characterise the baseline (undamaged) state of a structure.

From the sensor network, there are long sequences of data which can be regarded as existing in one of two main states: when the bridge is under load (train passage events) and under no load. In reasoning about deterioration, one might be interested in how quickly the bridge recovers after a train passage event. A tool is provided for extracting the train passage events from such data (see later in Section 3.2). To monitor the bridges' instantaneous health, models that handle the high frequency of the data are needed. These models can then be deployed for anomaly detection to identify departures from the recent historical behaviour, as illustrated in Section 3.6. Identifying the timing and frequency of such anomalies will provide another mechanism for reasoning about degradation. This long-term degradation through the sensor system is monitored. The data are the response of the sensor system to stimulus (in this case the passage of a train over the bridge) and not the response of the bridge itself. Thus, through the data, one is reasoning about the recovery of the sensor network and indirectly the bridge.

While there have been advances in recent years which have studied the application of statistical techniques in structural health monitoring (SHM), there is still significant scope for improvement and for introducing new concepts. Studies by Gul and Catbas (2009) investigated the use of autoregressive (AR) models in conjunction with an outlier detection algorithm based on the Mahalanobis distance. They validated their techniques based on two simplified laboratory steel beam and steel grid test specimens and under controlled ambient conditions. Rosales and Liyanapathirana (2017) investigated data obtained for a wireless sensor network attached to an experimental test frame. They employed both AR models and AR models with exogenous inputs (ARX) after the paper of Lei et al. (2003). Based on a comparison between the two techniques, they concluded that the ARX model, while being more computationally costly, provided significant improvement over the AR model in its potential to localise and quantify damage better. A study conducted by Noman et al. (2012) also utilised AR but applied the technique to a real structure, the Portage Creek Bridge in Victoria, Canada. They were able to use such techniques to conclude that little evidence of long-term deterioration was occurring within the structure. Another approach based on generalised Bayesian dynamic linear models (BDLMs) was proposed by Goulet (2017). Based on simulations, this study developed a framework for constructing, learning and estimating BDLMs whereby hidden effects such as daily and seasonal temperature variations and missing or outlier data could be incorporated.

While several previous studies have investigated various methods for modelling and interpreting data gathered from SHM systems, few have considered this challenge in the context of big data sets obtained from real structures and operating in real time. 'Selfsensing' or 'sensory' structures are those which contain an integrated sensor system for determining the state of the structure itself (Measures et al., 1992). Based on operational data gathered from a recently constructed self-sensing railway bridge, this study proposes several solutions for batch and real-time processing of the data. In particular, the primary research contributions from this paper include

- development of a statistical method based on adaptive linear models for analysing and interpreting large and continuously updated data sets in real time

- introduction of a real-time anomaly detection scheme based on individual and network sensor data.

\section{Self-sensing railway bridge}

\subsection{Sensor system}

Completed in March 2016, a 26.8 m composite steel-concrete half-through railway bridge located in Staffordshire, UK, was instrumented during its construction with a network of 134 fibre- 
Real-time statistical modelling of data generated from self-sensing bridges Lau, Butler, Adams, Elshafie and Girolami

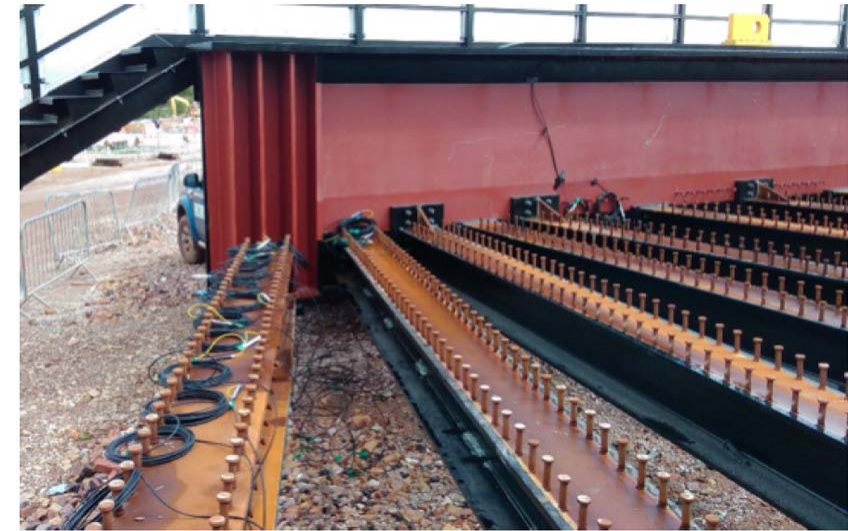

Figure 1. Installation of fibre-optic sensors on a bridge

optic strain sensors (FOSSs) (see Figure 1). The FOSS used in this study are based on Bragg gratings (fibre Bragg gratings or FBGs) which represent periodic changes in the index of refraction which can be inscribed at discrete points along the length of an optical fibre. As the FOSS cable and inscribed FBG are strained, the initially inscribed Bragg wavelength shifts and can be converted to an equivalent strain through a photoelastic coefficient. In addition to strain from mechanical effects (i.e. weight of passing trains etc.), FBGs are sensitive to changes in temperature particularly in how it affects their index of refraction and due to the thermal expansion of the optical fibre itself. Therefore, when evaluating measurements taken by FBGs over periods of time whereby significant temperature changes occur, appropriate temperature compensation techniques must be applied. The use of FBGs in the sensing system was chosen for their improved accuracy, reliability and resistance to corrosionbased deterioration. In addition, up to 20 individual FBG sensors can be inscribed along a single optical fibre, thereby greatly reducing wiring lengths and the number of interrogation channels. The FBGs installed as part of the SHM system were manufactured in low-bend-loss fibre with an additional glassfibre-reinforced polymer coating for added robustness during installation and operation. The FBGs along the optical fibre had inscribed Bragg wavelengths between 1510 and $1586 \mathrm{~nm}$ with an approximate strain accuracy of \pm 4 microstrain.

FBGs were installed and measurements were recorded throughout the construction phase. Critical superstructure elements including the two main I-girders, the midspan cross-beams, the midspan section of the reinforced-concrete deck and the midspan vertical web stiffeners on the east main girder were instrumented. In addition, three pre-stressed concrete sleepers were manufactured with several FBGs installed along the top and bottom prestressing strands at the rail seat locations and at their midspan. These self-sensing sleepers were installed at the midspan of the bridge to correspond to the location of the instrumented crossbeams. An overview of the monitoring system is presented in Figure 2 .

\subsection{Monitoring programme}

The monitoring programme has been divided into two phases: one during construction and the other during operation. Originally, the primary monitoring objectives included (a) evaluating the robustness of the sensor network during construction, (b) establishing a comprehensive pre-operational performance

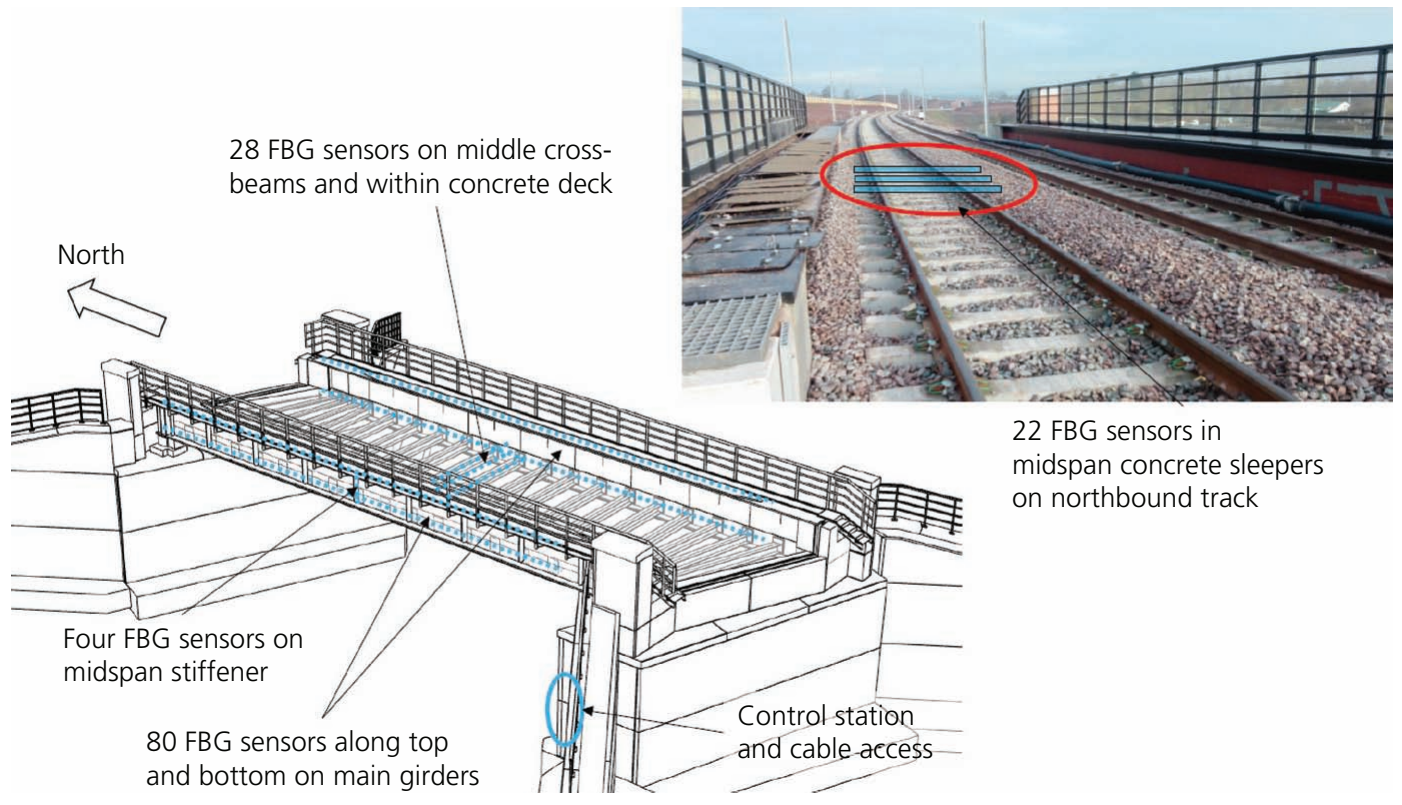

Figure 2. Fibre-optic-based monitoring system 


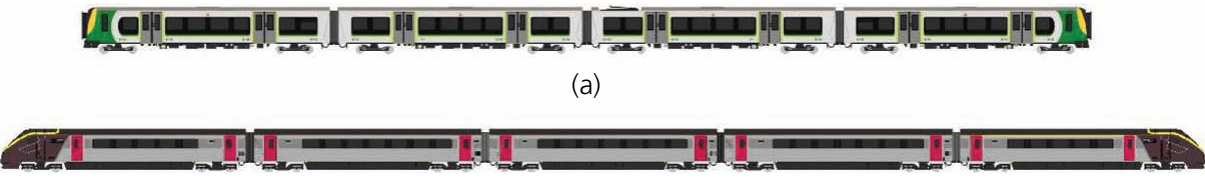

(b)

Figure 3. Typical train types: (a) class 350 Desiro; (b) class 221 Super Voyager

baseline and (c) developing analytical tools for long-term assessment, detection of damage (deterioration and/or anomalies) and management of self-sensing bridges. The first two objectives were previously addressed by Butler et al. (2016a).

Operational data on the self-sensing bridge have been recorded since July 2016, several months after the bridge was opened to passenger trains. Since then, strain readings for all of the 134 FBG sensors have been recorded during the passage of over 140 trains. The sensing system is capable of recording data continuously at $250 \mathrm{~Hz}$. The available data, which include 140 train passage events and a period inactivity around the event, consist of more than 24000000 strain readings. Depicted in Figure 3, two train types typically pass over the bridge, a British Rail class 350 'Desiro' (four-car formation) and a class 221 Super Voyager (four- or five-car formation). These different types of trains cause different responses in the sensor network.

\section{Statistical analysis and modelling}

This section provides a brief description of the sensor data and presents efficient batch methods for extracting train passage events from large data sets.

The extraction of train passage events into a database is a necessary precursor to reasoning about degradation. Studying the historic response of the sensor network when a train passes and its recovery will provide a benchmark to compare against when reasoning about degradation.

An efficient streaming procedure for modelling sensor data while they are being collected at $250 \mathrm{~Hz}$ is presented. The modelling procedure is used to address ambient (i.e. temperature) variations. Based on this streaming model, a method for tracking long-term deterioration (i.e. damage and anomaly detection) is introduced. The streaming model does not directly measure long-term deterioration but provides a way of detecting more immediate changes in the sensor network to extract train passage events. Later, in Section 4, it is discussed how to use these models to reason about future damage.

The distinction between batch and streaming is as follows. A batch procedure operates on a block of historic data which can be stored in memory, and the procedure is able to pass repeatedly over the data. In contrast, a streaming procedure updates when new data arrive and, due to computational constraints, can access the datum only once. Moreover, a streaming procedure needs to handle unknown temporal variation - that is, the phenomenon that the future will be different from the present for unknown reasons.

\subsection{Sensor data}

The sensor system consists of 134 fibre-optic sensors located at different positions on the bridge. Each fibre-optic sensor records wavelength over time, which measures horizontal strains at discrete locations on the bridge superstructure. As noted earlier, each sensor collects data at a rate of $250 \mathrm{~Hz}$. Figure 4 displays data collected from a single sensor showing two distinct states: the first is the train passage event highlighted in grey, and the second is the unloaded state of the bridge. A distinct feature of the data is the banding pattern which arises from the preprocessing algorithm implemented by the fibre-optic analyser. Figure 4 presents all the data, although it seems that fewer than 250 datapoints are shown every second - this is a display artefact.

The wavelength records can be converted to strain records as follows: Denote the wavelength at time $t$ as $\lambda_{t}$, then the strain at $t$ is

1. $\varepsilon_{t}=\frac{1}{1-\rho}\left(\frac{\lambda_{t}-\lambda_{1}}{\lambda_{1}}\right)$

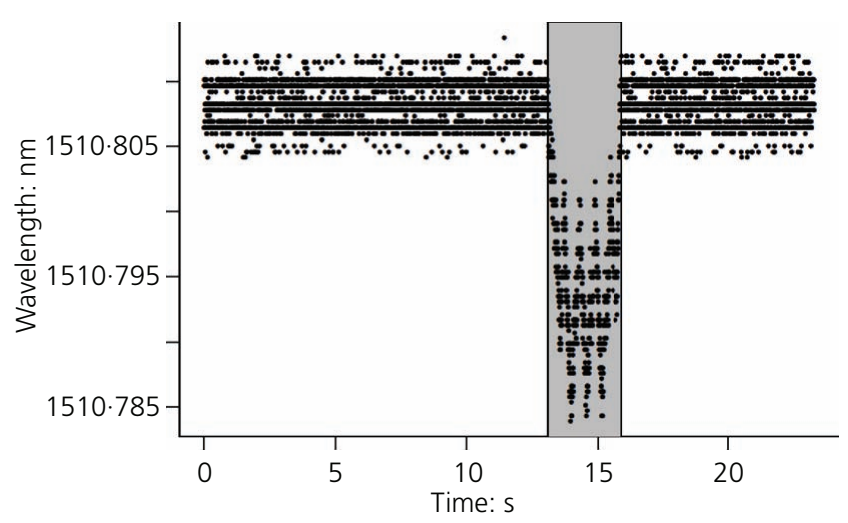

Figure 4. Data from a single sensor include a single train passage event highlighted by the grey region 
where $\rho=0 \cdot 22$ is the photoelastic coefficient. More precisely, note that the strain is the change in strain relative to the first reading. In the following sections, the methods and models will use strain.

\subsection{Batch processing of large data sets}

This section presents a procedure that extracts the train passage events from large data sets consisting of many sensor records. Figure 5 presents the strain records from a single sensor from the top of the east main girder. Although the record length is only (approximately) $40 \mathrm{~min}$, there are 611108 datapoints for this single sensor. Considering the entire network of 134 simultaneously recording sensors, this corresponds to over 81 million datapoints - which certainly represent a big data problem. The four pronounced spike features, highlighted by the grey dashed boxes in Figure 5, are train passage events. A method that automatically extracts these events, using the data in Figure 5 as a running example, is now introduced. The pseudocode for this procedure is presented in the Appendix.

As a first step, the main temporal variation in the data, which is likely due to variations in temperature during the data collection period, is removed. This temporal variation is estimated using the average of the data in a sliding window using $w=25000$ datapoints $(100 \mathrm{~s})$. The moving average is represented by the grey solid line in Figure 5. This moving average is subtracted from the strain data, which are then rescaled (see the Appendix for details) the result is presented in Figure 6. The train passage event times can now be identified by their large variation in comparison with the background data. To quantify the variation, the standard deviation of the detrended data is computed in a batch fashion. This is accomplished by dividing the data into non-overlapping batches of length $v=500$ datapoints. Then, the standard deviation for each batch is computed. A threshold of $\gamma=1.5$ microstrain is selected, such that a batch standard deviation above this threshold flags a train passage event. This procedure is repeated over all sensors. An

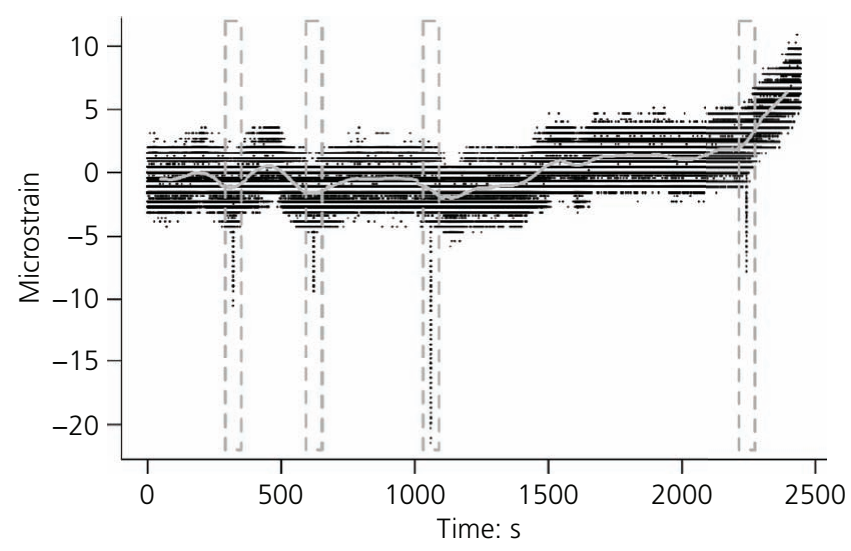

Figure 5. Data (black points) from a single sensor converted to strain. Moving average (grey solid line) captures the global trend of the data. The train passage events are highlighted in the grey dashed boxes

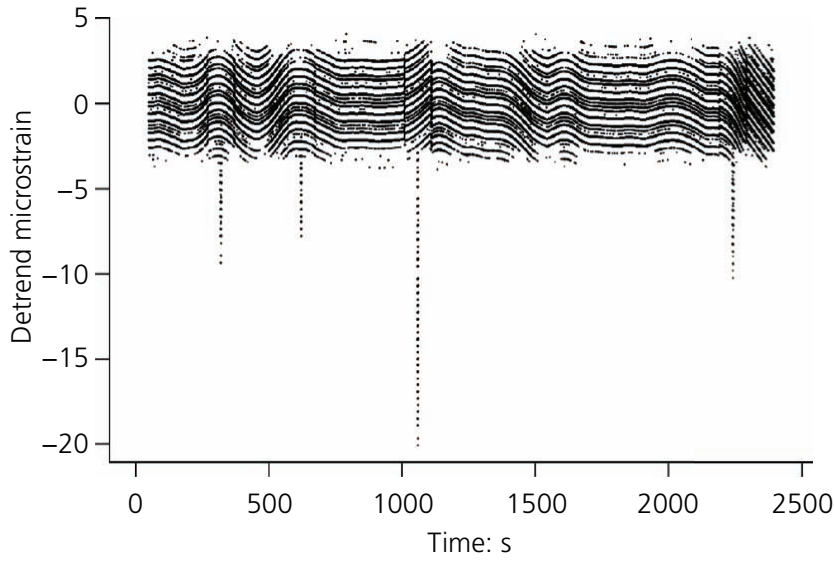

Figure 6. Detrended sensor data for removing temporal variation

alternative approach could be to treat the measurements across all sensors as a multivariate observation. The advantage of the procedure outlined earlier is its computational speed.

The outcome of this sensor-based procedure is a table of flagged events from each sensor with the number of sensors which suggested it (see Table 1). Notice that some of the train passage events times are within several seconds of each other. This is due to the delayed train response over the distributed sensor network or the peaks produced by the individual axles. This set of times is reduced using the following procedure. Any times that are within $2 \mathrm{~s}$ of each other are merged (see Table 2 for the result) since it is known that two train passage events cannot occur within this period. This knowledge is based on the average train speed, train lengths and bridge length. These times are then used to isolate the individual train passage events. For instance, the event at time 321 can be extracted by cutting around the event time from time $321 \pm 5 \mathrm{~s}$.

This is a computationally efficient procedure for extracting train passage events from batch data. An example of an extracted event is presented in Figure 7. In Section 4 the choice of control parameter values, $w, v$ and $\gamma$, is discussed.

\subsection{Statistical modelling}

This section discusses how to monitor sequentially the sensor strain readings individually and collectively using a statistical model. In

Table 1. Train passage event times across 134 sensors using extraction method

$\begin{array}{llllllllll}\text { Time: } \mathrm{s} & 320 & 322 & 622 & 624 & 1060 & 1062 & 2244 & 2246 & 2248 \\ \text { Count } & 121 & 115 & 117 & 95 & 100 & 103 & 123 & 111 & 98\end{array}$

Table 2. Merged train passage event times across 134 sensors using the extraction method

$\begin{array}{lllll}\text { Time: } \mathrm{s} & 321 & 623 & 1061 & 2246 \\ \text { Count } & 236 & 212 & 203 & 332\end{array}$




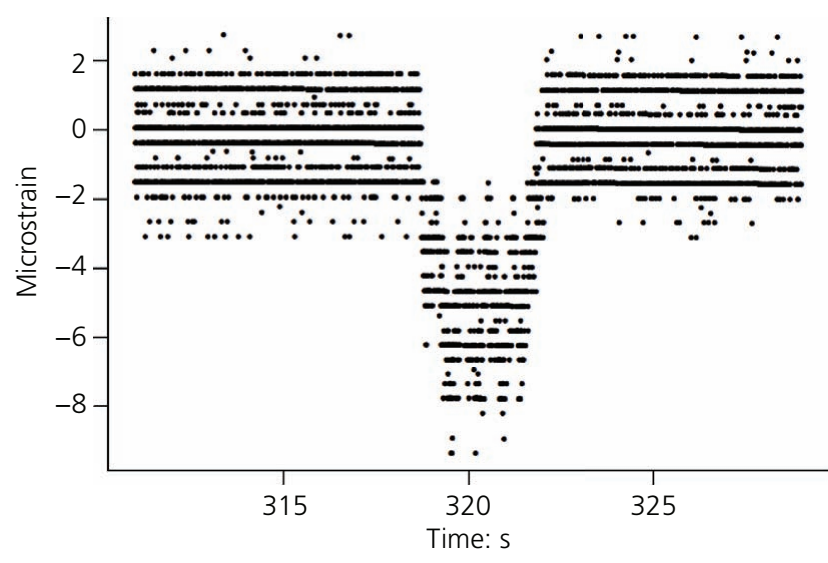

Figure 7. Extracted train passage event from a single sensor

constructing such a streaming model, considerations need to be taken that address issues of computational efficiency, to handle data arriving at $250 \mathrm{~Hz}$; adaptation over time, to account for the temporal variation (i.e. due to temperature effects) and data storage.

Denote the strain record for sensor $s$ at time $t$ as $Y_{t}^{(s)}$. Further, denote the number of sensors as $S$. For sensor $s$, the strain is modelled as

$$
\begin{aligned}
M_{s}: & Y_{t}^{(s)} \\
& =\beta_{0}+\sum_{u \in(1, \ldots, S) \mid s} \beta_{u} Y_{t-1}^{(u)}+\omega_{t} \quad \omega_{t} \sim N\left(0, \sigma^{2}\right)
\end{aligned}
$$

for $t=2,3, \ldots$ and where $N\left(\mu, \sigma^{2}\right)$ denotes a normal distribution with mean $\mu$ and variance $\sigma^{2}$. The model $M_{s}$ is a linear model that describes the strain measurements from sensor $s$ at time $t$ as a linear combination of all other sensor measurements at time $t-1$. Notice that this model is a one-step-ahead forecast for sensor $s$, without using sensor $s$ information. Models of the form of $M_{s}$ are used for each sensor, primarily for computational speed in sequential updating settings. It is shown later that this model describes strain measurements from sensor $s$ without using sensor $s$ data, providing surprisingly accurate predictions.

The unknown parameters of $M_{s}$ are $\beta_{j}$ and $\sigma^{2}$. In batch settings, these parameters are typically estimated using maximum likelihood or equivalently a least-squares method. Fortunately, the linear structure of these models admits efficient sequential updating and allows the inclusion of a parameter called a forgetting factor which provides temporal adaptation.

\subsection{Updating the model}

At a particular time $t$, only certain information has been revealed, namely, $\left\{Y_{\tau}^{(s)}: \tau=1, \ldots, t ; s=1, \ldots, S\right\}$. Refitting model $M_{s}$ when new measurements are received is impractical due to the high data acquisition rate (250 measurements per second). Moreover, it would be undesirable to have a growing window of data due to temporal variation (see Figure 5), and using a sliding window is to be avoided. Therefore, a recursive method to update the model parameters is used. This updating of linear models is called recursive least squares (see chapter 9 in the book of Haykin (2002)). This procedure will update the model parameters faster than the acquisition of new data (discussed later in Section 3.8). Further, this streaming regression has fixed computation and memory demand and requires that no data need be stored.

\subsection{Forgetting factor}

To account for the temporal adaptation in the data, a forgetting factor, $\lambda_{f} \in(0,1)$, is introduced into the model $M_{s}$, which effectively puts more weight on recent data during the updating procedure. This approach was proposed chapter 9 of the book by Haykin (2002) and provides both temporal adaptation and efficient updating. For the purpose of exposition, a single fixed $\lambda_{f}$ value is used, although it is possible to tune sequentially (e.g. see the paper of Anagnostopoulos et al. (2012)).

The concept of the forgetting factor is now illustrated using a simple example. Consider computing the average for a sequence of values $x_{1}, x_{2}, \ldots$ (for instance, strain measurements). The following are the recursive equations for updating the average value of $x_{1}, x_{2}, \ldots$ with and without a forgetting factor $\lambda_{f}$. The average of the data at time $t$ is denoted as $\bar{x}_{t}$ and $m_{0}=n_{0}=0$

without the forgetting factor

$$
\begin{aligned}
m_{t} & =m_{t-1}+x_{t} \\
n_{t} & =n_{t-1}+1 \\
\text { 3. } \bar{x}_{t} & =\frac{m_{t}}{n_{t}}
\end{aligned}
$$

with the forgetting factor

$$
\begin{aligned}
m_{t} & =\lambda_{f} m_{t-1}+x_{t} \\
n_{t} & =\lambda_{f} n_{t-1}+1 \\
\text { 4. } \quad \bar{x}_{t} & =\frac{m_{t}}{n_{t}}
\end{aligned}
$$

Note that $\lambda_{f}=1$ has no temporal adaptation, whereas as $\lambda_{f}<1$ downweight the older data. The $n_{t}$ is a value that, loosely speaking, describes the number of datapoints used in computing the average, akin to the effective sample size. This simple concept readily transfers to updating the parameter estimates of the linear model $M_{s}$.

So far, a statistical tool that can sequentially and adaptively predict the one-step-ahead reading for sensor $s$ given the previous tick of data from other sensors has been introduced. That is, the model provides a point estimate of the strain measurement of sensor $s$ at time $t$. Figure 8 presents these point estimates and the true measurements from a single sensor. Before turning to the construction of an anomaly detection method, which requires a 
Real-time statistical modelling of data generated from self-sensing bridges Lau, Butler, Adams, Elshafie and Girolami

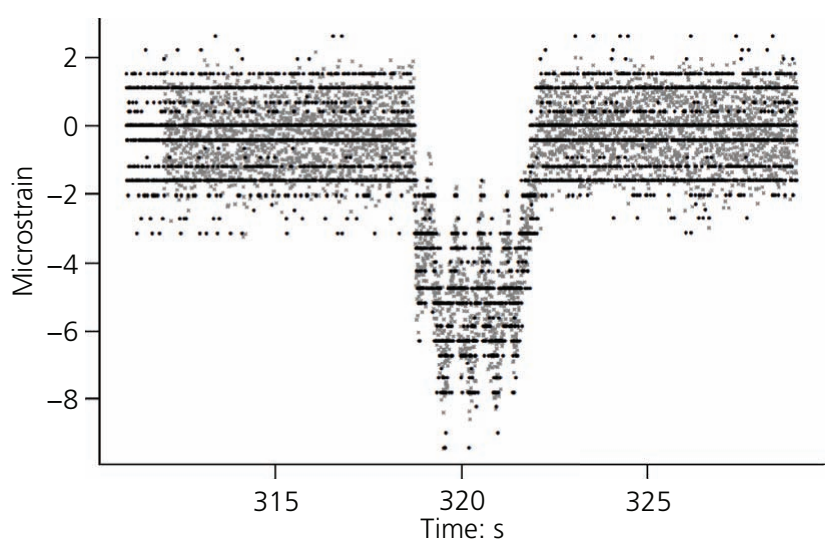

Figure 8. Predicted strain values for sensor 1 using model $M_{1}$ with $\lambda_{f}=0.99$. Black points represent the observed measurements, and grey cross points represent the predicted point estimated values from the model

measure of the uncertainty of the estimate, a statistic used to quantify the difference between the point estimate and the observed data is introduced.

A modified $R^{2}$ statistic, the coefficient of determination, that monitors the goodness of fit of a model is computed. This statistic measures how much variation in the data from sensor $s$ is explained by the regression model. This version of the $R^{2}$ statistics slightly differs from the coefficient of determination commonly used with linear models, as it incorporates the forgetting factor. The modified $R^{2}$ statistic for model $M_{S}$ is

5. $R^{2}=\frac{\sum_{t} \lambda_{f}^{n-t}\left(Y_{t}^{(s)}-\widehat{Y}_{t}^{(s)}\right)^{2}}{\sum_{t} \lambda_{f}^{n-t}\left[Y_{t}^{(s)}-(1 / t) \sum_{k=1}^{t} Y_{k}^{(s)}\right]^{2}}$

where $\widehat{Y}_{t}$ is the prediction of $Y_{t}$.

Figure 9 presents the modified $R^{2}$ statistic computed for the streaming model using $\lambda_{f}=0.99$. Two features are notable in Figure 9. First, the model provides a reasonably good fit throughout the observation period, indicated by the high $R^{2}$ values. Second, during the time of the train passage event, the model becomes increasingly accurate at predicting the sensor measurements (represented by $R^{2}$ values close to 1 ), indicating that the sensor readings move to a state of an even higher correlation.

In Figure 9, the $R^{2}$ values after the train passage event do not return to the values prior to the event. There are two plausible explanations for this. First, it takes the sensor network and bridge longer to recover than the observation period. Second, the choice of a fixed forgetting factor, $\lambda_{f}=0.99$, is suboptimal in respect to the estimation between different regimes. As noted earlier, this can be alleviated by using sequential methods for tuning of the forgetting factor.

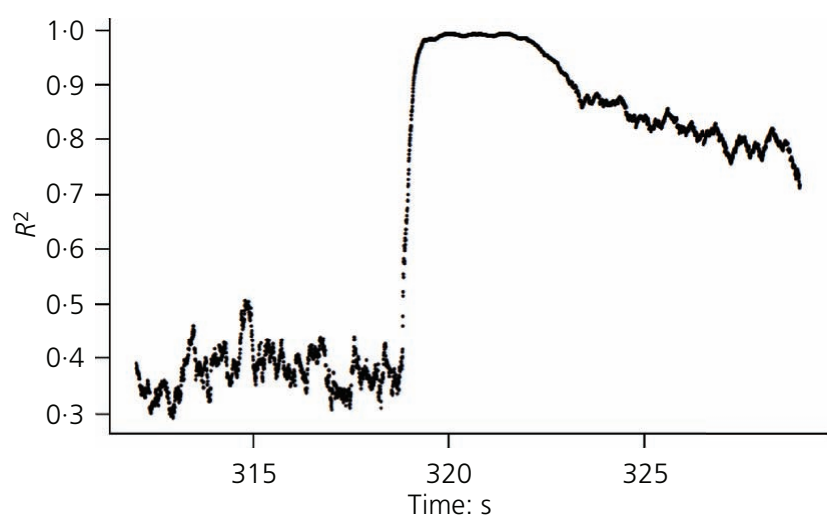

Figure 9. Modified $R^{2}$ statistic for model $M_{s}$ with $\lambda_{f}=0.99$

\subsection{Individual sensor changes}

Based on the developed statistical model, an anomaly detection method is constructed through characterisation of the models' predictive uncertainty. This involves computing a $p$-value (or constructing a prediction interval) for the next datapoint and flagging the datapoint as unusual if falls below a given threshold. This procedure is first applied to a single sensor and then extended to the collective sensor network.

The theory of linear models is used to construct a $p$-value which extends to the context of the developed streaming regression, provided that the forgetting factor $\lambda_{f}$ does not depend on the data. The core result is

6. $Y_{t}^{(s)} \sim N\left[\widehat{Y}_{t}^{(s)}, \operatorname{var}\left(\widehat{Y}_{t}^{(s)}\right)\right]$

where $\widehat{Y}_{t}^{(s)}$ is the one-step-ahead prediction for sensor $s$ at time $t-1$. From this result, a $p$-value, $p_{s}$, can be computed. This is a measure of how surprising the new datapoint is with respect to the model. Figure 10 shows the sequence of $p$-values for a specific

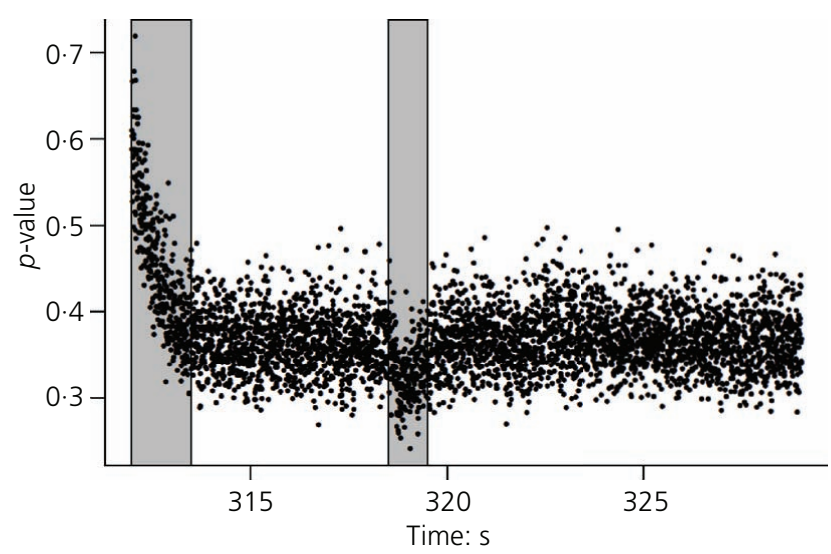

Figure 10. Sequence of $p$-values from $M_{s}$ using $\lambda=0.99$ 
sensor. The far left side, highlighted by the grey area in Figure 10, relates to the initialisation of the model, after which reasonable parameter estimates are obtained. Further, the decrease in $p$-values, highlighted by the central grey area in Figure 10, indicates the response of the sensor to a passing train.

To construct an anomaly detection method, the $p$-value, $p_{s}$, is compared with a threshold $\alpha$, such that if $p_{s}<\alpha$, an anomaly is flagged. The choice of $\alpha$ is determined by the required detection sensitivity of the system. Any such statistical procedure will make mistakes by chance, and $\alpha$ is set to balance this false positive rate with the amount of detections that are of practical interest. Since hundreds of tests per second are being performed, the threshold $\alpha$ should be selected to be very small. Performing multiple tests will inevitably lead to a high false signal rate.

A flagged anomaly is the departure from the new observation from the postulated model $M_{s}$, which is based on the sensor measurements. This flagged anomaly could relate to the failure of the sensor - that is, debonding of the sensor from the structure and/or damage of the bridge at the sensor location. Therefore, this anomaly detection method can be used to indicate locations on the superstructure where individual sensors may be faulty and/or elements of the structure are damaged.

\subsection{Sensor network changes}

In Section 3.6, an anomaly detection method for individual sensors was introduced, where a sequence of $p$-values for each sensor was produced. This anomaly detection method is capable only of flagging anomalies in individual sensors. From each individual sensor model, the $p$-values can be efficiently computed, and, indeed, models for all sensors can be computed at the same rate (or faster) as the data acquisition rate (i.e. $250 \mathrm{~Hz}$ ). To monitor the global response of the bridge, the $p$-values from all sensors are combined to provide an overall view of the collective sensor system response. Combination of $p$-values is well studied in statistics and Fisher's (1925) method is a popular approach. Under the null hypothesis that each model $M_{S}$ is the true data generating model, Fisher's method uses the statistic

7. $X^{2}=-2 \sum_{s=1}^{S} \log \left(p_{s}\right)$

This $X^{2}$ follows a $\chi_{2 S}^{2}$ distribution under the null hypothesis.

Fisher's method is motivated to combine independent $p$-values. While this is not true in this sensor network setting, the modifications required for dependent $p$-values requires knowledge of the dependence structure.

Figure 11 shows the $X^{2}$ scores for the 80 FBG sensors installed along the top and bottom flanges of the east and west main girders, for the data extracted in Section 3.2. These scores would

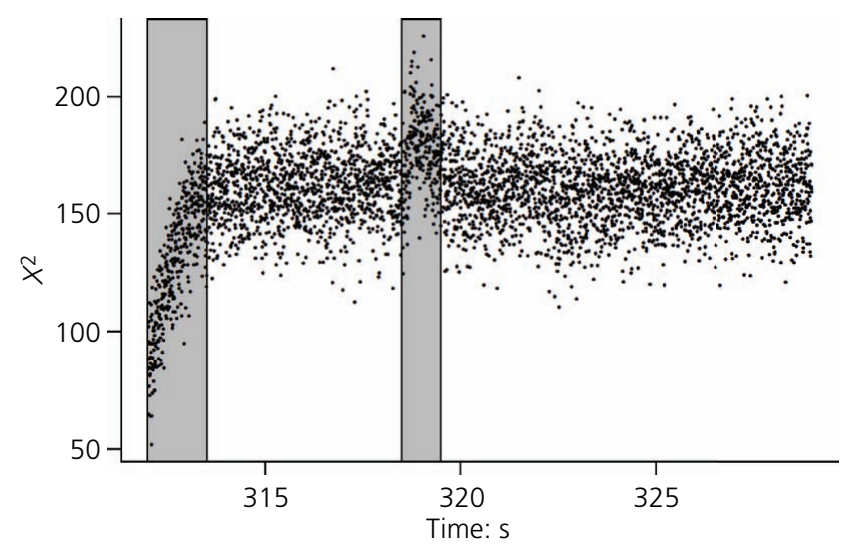

Figure 11. $X^{2}$ scores from 80 sensors located in the girders

again require comparison with a threshold to flag anomalies. In examining the $X^{2}$ values in Figure 11, the train passage event is seen (highlighted by the central grey box). As discussed in Section 3.6 for the individual sensor $p$-values, the far left side relates to the initialisation of the model. The unloaded periods of the data are $\chi_{2 S}^{2}$ distributed as indicated by Fisher's method. This $X^{2}$ statistic provides a collective summary of the response from the entire sensor network. As with the individual $p$-values, an anomaly detection method would require the $X^{2}$ score to be compared with a threshold, $\zeta$, such that if $X^{2}>\zeta$, an anomaly would be flagged. A flagged anomaly in this case would suggest a collective change in all the sensors. Such an anomaly detection method can used to indicate problems with the entire sensor network and/or the whole bridge.

\subsection{Speed of computation}

The previous sections introduced a streaming linear model which sequentially and adaptively updates its parameter estimates, enhanced with a detection framework to flag changes in individual sensors and the entire sensor network.

To be practically useful, the model updating process and $p$-value computation must take less time than the arrival between two consecutive strain records. This study's computations, done on an off-site $1.7 \mathrm{GHz}$ laptop computer, show that updating a single model with a new observation and computing the $p$-value takes on average $2.3 \times 10^{-4} \mathrm{~s}$ (with a standard deviation of $4 \times 10^{-6} \mathrm{~s}$ ), which is faster than the $250 \mathrm{~Hz}$ data rate. Parallelisation of the entire updating procedures across each model is possible, and the additional effort of computing the $X^{2}$ statistic is negligible. The statistical software $\mathrm{R}$ was used in this study to perform the computations. Implementations of these methods in other programming languages such as $\mathrm{C}++$ would lead to a significant increase in computation speed.

\section{Results and discussion}

The previous sections have presented a number of useful processing methods and statistical models for extracting critical 
structural response information provided by a fibre-optic sensor network installed on a newly constructed railway bridge. Based on the developed models, a framework for tracking statistically significant changes in the individual sensors as well as across entire groups of sensors was presented. While the data set considered in this study represents a relatively small sample of the total amount of data currently being generated from the selfsensing bridge, the statistical techniques developed are directly applicable to any size of data set. This is important for the longterm monitoring of structures in which many months and years of sensor data may be required to be analysed and assessed. The following sections discuss additional considerations for statistical modelling, provide insight into how these techniques may be used in long-term SHM and discuss the applicability of the developed techniques to other structure types.

\subsection{Statistical modelling}

The statistical model and the anomaly detection methods provide the basis of a monitoring system capable of providing real-time updates on the bridges' sensor network health. There are a number of control parameters in the methods proposed throughout this work. In the processing of a large data set (Section 3.2), the parameters are the width of the sliding window, $w$; the length of the non-overlapping batches, $v$ datapoints; and the standard deviation threshold, $\gamma$. The values used in Section 3.2 lead to the extraction of all the train passage events from big data sets. For other applications - for example, where the event signal is not distinct - the parameter values may need to be tuned. The forgetting factor, $\lambda_{f}$, used in the statistical model (Section 3.3) is another parameter which needs to be chosen. This parameter can be tuned using past data, however, the corresponding theoretical results, used to construct the anomaly detection method, no longer hold. Moreover, this tuning would require further computational effort. The anomaly detection methods outlined in Sections 3.6 and 3.7 both require a threshold to be set in some fashion. For instance, for the collective sensor network summary discussed in Section 3.7, a cumulative sum (cusum) chart (Page, 1954) can be used to monitor the $X^{2}$ scores and signal a change. Cusum charts can be adapted to detect a change - for example, a shift of the mean - in the distribution of a sequence of $X^{2}$ scores. The cusum chart methodology lends itself particularly well to this problem since it is a sequential method with quick updates.

\subsection{Long-term SHM}

Traditionally, bridge condition monitoring and assessment is performed on the basis of visual condition surveys to provide a condition rating for the bridge. Self-sensing bridges allow for a data-driven approach to condition monitoring where assessment of a bridge's health can be based on the data gathered through the sensing system. The monitoring of the sensor network, discussed in Section 3.7, can be used to study groups of sensors - for example, west against east main girders - in order to assess their long-term load sharing ratio. These types of statistical modelling and anomaly detection methods may be used to form the basis of an SHM system. For instance, if the $X^{2}$ scores which characterise the response of the sensor network (see Section 3.7) deviate from its known null distribution while the bridge is unloaded, then some global structural change may have occurred. Another way to monitor the structural health of the bridge is to compare similar train passage events (extracted by the method outlined in Section 3.2) for changes. For similar trains - that is, same number of carriages, similar mass and so on - it would be expected that the bridge and sensor response be almost identical. Therefore, significant changes in the bridge/sensor response may indicate alteration in the structure.

Applying statistical techniques to long periods of data allows for the ability to characterise the effects of environmental factors (e.g. temperature and humidity) on the structural response of the bridge. These characterisations will enable more accurate models to be developed which will provide better measures of how the bridge deteriorates with time. If real-time processing of certain sensor data sets is not critical, implementation of other statistical techniques which are capable of damage identification and localisation is also possible. Ideas for addressing these issues, from a statistical standpoint, are discussed in the paper of Lau et al. (2018) and form the basis of the authors' future work in this area.

\subsection{Other sensor and structure types}

The discussion up to this point has focused on extracting information from and applying statistical techniques to strain data. However, it is worth noting that the strain data themselves may be pre-processed in order to calculate other measures important for assessment of structural condition. These measures could include beam curvature, stresses and neutral axis location, all of which could be modelled, tracked over time and used as indicators of long-term deterioration. Therefore, the techniques presented earlier may also be directly applied to other structural performance measures, including strain. Data collected from other sensor types installed on a structure which continuously measure displacement, acceleration, temperature and so on can also be readily assessed and analysed using the proposed statistical methods. Steel-composite bridges are not the only structures which have been instrumented with permanent monitoring systems; for instance, another railway bridge composed of prestressed concrete girders and a composite concrete deck slab has also been instrumented with an integrated fibre-optic sensor network and is currently being studied by Butler et al. (2016b). In addition, a variety of other structures reported in the literature, including high-rise buildings (Glisic et al., 2005), tunnel linings and reinforced-concrete foundation piles (Kechavarzi et al., 2016), have all implemented continuously recorded sensing systems. A variety of self-sensing structures, in which large sets of continuously collected data are required to be processed and analysed efficiently and expediently, can leverage the statistical techniques presented herein.

\section{Conclusion}

This paper presents a big data case study involving a self-sensing railway bridge which has been instrumented with 134 discrete 
fibre-optic sensors which record strain simultaneously at $250 \mathrm{~Hz}$. A subset of the overall bridge monitoring data set has been used in order to develop statistical tools which can process and analyse the data while being continuously updated. A new processing method for extracting useful operational information from long periods of sensor records was first presented. This fast, batch method extracts the individual train passage events within the large data sets and decouples the underlying background strain changes due to environmental effects such as temperature change. The extraction method will be of great practical use to engineers and operators who are tasked with quickly processing large sensor data sets generated from self-sensing bridges.

A recursive statistical model was then introduced that is able to update faster than the incoming recorded data, adapt to account for the temporal variation (i.e. due to environmental effects) in the data through implementation of a forgetting factor and accurately describe the data over time while requiring only minimal data storage. Based on these adaptive models, anomaly detection methods were developed and are capable of monitoring sensors individually (based on $p$-values) and across the entire sensor network (based on $X^{2}$ statistic). The $X^{2}$ scores which characterise the response of a group of sensors (and a component of the bridge) can be tracked over time for any deviations from their baseline distribution in order to provide an indication of deterioration and/or damage.

The statistical tools developed as part of this study will form the core component of a long-term SHM strategy in which considerations such as weekly, seasonal and yearly environmental trends can be characterised and used to update and create more robust prediction models. In addition, techniques developed in this study may be directly implemented in the analysis of other measured quantities (e.g. displacement, acceleration, temperature) and structure types (e.g. high-rise buildings, tunnels). This combination of a real-world self-sensing bridge case study and an innovative statistical framework for efficiently analysing the large monitoring data sets provides a valuable demonstrator for smart infrastructure systems.

\section{Acknowledgements}

The authors would like to acknowledge the Lloyd's Register Foundation, the Engineering and Physical Sciences Research Council (EPSRC) and Innovate UK for funding this research through the Programme on Data-centric Engineering at the Alan Turing Institute and through the Centre for Smart Infrastructure and Construction Innovation and Knowledge Centre. Research related to installation of the sensor system was carried out under EPSRC grant number EP/L010917/1. Data related to this publication are available at the University of Cambridge data repository (University of Cambridge, 2018).

\section{Appendix: Algorithm for train passage event extraction}

Data: Denote the strain measurement from sensor $s$ at time $t$ as $Y_{t}^{(s)}$ for $s=1, \ldots, S$ and $t=1, \ldots, T$.

Input: Moving average length $w$; Batchlength $v$ datapoints; Standard deviation threshold $\gamma$.

Output: Sensor $s$ 's train passage event times, $U_{\mathrm{s}}$, for $s=1, \ldots, S$.

for $s=1,2, \ldots, S$ do

Compute moving averages

(8)

$Z_{b}=\frac{1}{2 k+1} \sum_{j=-k}^{k} Y_{b+j+1}^{(s)} \quad$ for $b=k, k+1, \ldots, T-k-1$

where $k=\lfloor w / 2\rfloor$ is the half-width of the sliding window ; Detrend the series

(9) $\widetilde{Y}_{b}^{(s)}=Y_{b}^{(s)}-Z_{b} \quad$ for $b=k, k+1, \ldots, T-k-1$

Scale the detrended series

(10) $C_{b}^{(s)}=\frac{\widetilde{Y}_{b}^{(s)}}{\sqrt{\frac{1}{2 l} \sum_{\tau=b-l}^{b+l}\left(\widetilde{Y}_{\tau}^{(s)}\right)^{2}}}$

where $l=\lfloor v / 2\rfloor$ is the half-width of the batches;

Compute the standard deviation in batches of length $v$

(11) $\sigma_{j}=\sqrt{\frac{1}{v-1} \sum_{i=(j-1)}^{j v}\left(C_{i}^{(s)}\right)^{2}}$

Identify large $\sigma j$

(12) $U_{S}=\left\{v j: \sigma_{j}>\gamma\right\}$

end

\section{REFERENCES}

Anagnostopoulos C, Tasoulis DK, Adams NM, Pavlidis NG and Hand DJ (2012) Online linear and quadratic discriminant analysis with adaptive forgetting for streaming classification. Statistical Analysis and Data Mining 5(2): 139-166, https://doi.org/10.1002/sam. 10151.

Bowers K, Buscher V, Dentten R et al. (2016) Smart Infrastructure: Getting More from Strategic Assets. Centre for Smart Infrastructure and Construction, University of Cambridge, Cambridge, UK.

Butler LJ, Gibbons N, Middleton C and Elshafie MZEB (2016a) Integrated fibre-optic sensor networks as tools for monitoring strain development in bridges during construction. The 19th Congress of IABSE Proceedings, Stockholm, Sweden, 21-23 September, pp. 1767-1775.

Butler LJ, Gibbons N, He P, Middleton C and Elshafie MZ (2016b) Evaluating the early-age behaviour of full-scale prestressed concrete beams using distributed and discrete fibre optic sensors. Construction 
Smart Infrastructure and Construction Volume 171 Issue SMIC 1
Real-time statistical modelling of data generated from self-sensing bridges Lau, Butler, Adams, Elshafie and Girolami and Building Materials 126: 894-912, https://doi.org/10.1016/j. conbuildmat.2016.09.086.

Fisher R (1925) Statistical Methods for Research Workers. Oliver and Boyd, Edinburgh, UK.

Glisic B, Inaudi D, Lau JM, Mok YC and Ng CT (2005) Long-term monitoring of high-rise buildings using long-gauge fibre optic sensors. 7th International Conference on Multi-purpose High-rise Towers and Tall Buildings, Dubai, UAE, 10-11 December.

Goulet JA (2017) Bayesian dynamic linear models for structural health monitoring. Structural Control and Health Monitoring 24(12): e2035, https://doi.org/10.1002/stc.2035.

Gul M and Catbas FN (2009) Statistical pattern recognition for structural health monitoring using time series modeling: theory and experimental verifications. Mechanical Systems and Signal Processing 23(7): 2192-2204, https://doi.org/10.1016/j.ymssp.2009. 02.013.

Haykin SS (2002) Adaptive Filter Theory. Prentice-Hall Information and System Sciences Series. Prentice Hall, Englewood Cliffs, NJ, USA.

Kechavarzi C, Soga K, deBattista N et al. (2016) Distributed Fibre Optic Strain Sensing for Monitoring Civil Infrastructure: a Practical Guide. ICE Publishing, London, UK.
Lau FDH, Adams NM, Girolami MA, Butler LJ and Elshafie MZEB (2018) The role of statistics in data-centric engineering. Statistics \& Probability Letters 138: 58-62, https://doi.org/10.1016/j.spl.2018.02.035.

Lei Y, Kiremidjian A, Nair K et al. (2003) Statistical damage detection using time series analysis on a structural health monitoring benchmark problem. Proceedings of the 9th International Conference on Applications of Statistics and Probability in Civil Engineering, San Francisco, CA, USA, pp. 6-9.

Measures RM, LeBlanc M, Liu K et al. (1992) Fiber optic sensors for smart structures. Optics and Lasers in Engineering 16(2): 127-152, https:// doi.org/10.1016/0143-8166(92)90005-R.

Noman AS, Deeba F and Bagchi A (2012) Health monitoring of structures using statistical pattern recognition techniques. Journal of Performance of Constructed Facilities 27(5): 575-584, https://doi.org/ 10.1061/(ASCE)CF.1943-5509.0000346.

Page ES (1954) Continuous inspection schemes. Biometrika 41(1-2): 100-115, https://doi.org/10.1093/biomet/41.1-2.100.

Rosales MJ and Liyanapathirana R (2017) Data driven innovations in structural health monitoring. Journal of Physics: Conference Series 842(1): 012012, https://doi.org/10.1088/1742-6596/842/1/012012.

University of Cambridge (2018) https://doi.org/10.17863/CAM.20380.

\section{How can you contribute?}

To discuss this paper, please email up to 500 words to the editor at journals@ice.org.uk. Your contribution will be forwarded to the author(s) for a reply and, if considered appropriate by the editorial board, it will be published as discussion in a future issue of the journal.

Proceedings journals rely entirely on contributions from the civil engineering profession (and allied disciplines). Information about how to submit your paper online is available at www.icevirtuallibrary.com/page/authors, where you will also find detailed author guidelines. 